\title{
¿Obesidad o desnutrición? Problema actual de los niños peruanos menores de 5 años*
}

\author{
Rosana Tazza ${ }^{1}$, Luz Bullón ${ }^{2}$
}

Resumen $\quad$ Objetivo: Determinar el estado nutricional de los niños peruanos menores de 5 años. Diseño: Evaluación de indicadores antropométricos. Lugar: Programa de Post Grado en Nutrición Pública, Universidad Nacional Agraria La Molina, y Departamento de Estadística e Informática. Materialy Métodos: Se analizó las bases de datos nacionales de las Encuestas Demográficas y de Salud Familiar (Endes), de los años 1991/1992, 1996 y 2000. Principales medidas de resultados: Desnutrición en niños menores de 5 años. Resultados: Durante el período evaluado, la desnutrición crónica disminuyó de 39\% a 32\%, la desnutrición global de 12\% a 9\% y la desnutrición aguda de $2 \%$ a 1\%. Mientras tanto, el sobrepeso y la obesidad ascendieron de 17\% y 5\% a 19\% y 7\%, respectivamente. La Endes 2000 muestra un promedio nacional de sobrepeso y obesidad en niños menores de cinco años de 25,19\%, siendo los mas afectados Tacna (57\%), Moquegua (42\%) y Lima (41\%); niños de 48 a 59 meses, extremadamente pobres y del área urbana. Según las proyecciones departamentales, Tumbes y Ucayali, a partir del año 2004, sobrepasarían el 50\% de sobrepeso. Las variables más influyentes para el desencadenamiento del problema son el nivel de pobreza, la edad del niñoy el área de residencia, descartándose el género, la instrucción de la madre yel departamento de proveniencia, como variables significativas. Conclusiones: Aunque persiste el problema de desnutrición en los niños peruanos, el sobrepeso y la obesidad vienen alcanzando valores considerablemente elevados, con proyecciones que van en rápido incremento.

Palabrasclave Trastornos nutricionales; obesidad; infante; estado nuticional.

Obesity or undernourishment? Current problem in less than five years old Peruvian children

\begin{abstract}
Objective: To determine the nutritional state in less than five years Peruvian children. Design: Evaluation of antropometric indicators. Setting: Post Graduate Program in Public Nutrition, La Molina Agriculture National University and Department of Statistics and Informatics. Material and Methods: Data bases of the 1991/1992, 1996 and 2000 Demographic Surveys and Familiar Health were analyzed(Endes). Main outcome measures: Nutrition in less than five year-old children. Results: Chronic undernourishment diminished from 39\% to 32\%, global undernourishment from $12 \%$ to $9 \%$ and acute undernourishment from $2 \%$ to $1 \%$ during the studied period. Overweight and obese children increased from $17 \%$ and $5 \%$ to $19 \%$ and $7 \%$,
\end{abstract}

Trabajo premiado con el segundo lugar en el Concurso Nacional de Tesis de Postgrado. ANR. Lima, Perú 2006.

1 Programa de Post Grado en Nutrición Pública, Universidad Nacional Agraria La Molina (UNALM). Lima, Perú

2 Departamento de Estadística e Informática, UNALM. Lima, Perú. respectively. According to Endes 2000, less than five years overweight and obese children national average was 25,19\%, most affected in Tacna (57\%), Moquegua (42\%) and Lima (41\%), 48 to 59 months children, extremely poor and the urban area. According to the departmental projections, Tumbes and Ucayali would exceed $50 \%$ of overweight in 2004. The most influential variables for the outbreak of the overweight children were poverty level, child age and area of residence, discarding the social status, education level of the mother and department provenience. Conclusions: Although the undernourishment problem persists in Peruvian children, the overweight and obese are reaching considerably high values with fast increase projections.

Key words: Nutrition disorders; obesity; child, preschool; nutritional status.

\section{INTRODUCCIÓN}

Anteriormente, un niño gordo significaba un niño sano, que podría sobrevivir los rigores de la desnutrición y de la infección. Sin embargo, en la última década, la gor- 
dura excesiva se ha convertido en un problema de salud primario de la niñez, por los efectos perjudiciales, tanto sociales como clínicos, que origina. Se considera entre otros la hipertensión arterial e hiperlipidemia, problemas ortopédicos relacionados con el peso (pie plano y escoliosis), desórdenes de piel, complicaciones psicológicas (inestabilidad emocional, conducta introvertida, autoestima baja) y hasta secuelas psiquiátricas potenciales, que ocasionan que los niños obesos constituyan una población de riesgo. Además, esta problemática conlleva a la mayor probabilidad de aparición de una serie de complicaciones en la adultez, como son las enfermedades crónicas no transmisibles, que incrementarán los riesgos de morbimortalidad, tanto a nivel nacional como mundial.

Se estima que, un tercio de los adultos con sobrepeso ya lo presentaba durante la niñez; y existen estudios que aseguran que la obesidad en estos casos es más severa que la que aparece en la edad adulta. Estas evidencias científicas son por sí solas razones de peso para combatir la obesidad desde la infancia. En Latinoamérica, el sobrepeso y la obesidad son de alguna manera subestimados, por ser considerados problemas propios de los países desarrollados y menos relevantes, ante los problemas de desnutrición existentes vinculados al deterioro socioeconómico de la población. Sin embargo, destaca el incremento de obesidad en países que presentan altos índices de pobreza y déficit de crecimiento, entre los que se considera el Perú, una sociedad caracterizada por la continua migración de población rural que genera una rápida urbanización, la cual a su vez influye en la problemática alimentaria, originando el doble problema de desnutrición y obesidad en el mismo hogar, caracterizando el fenómeno denominado 'transición nutricional'.

Desafortunadamente, en nuestro medio el sobrepeso y la obesidad ( $\mathrm{S}$ y $\mathrm{O}$ ) conti- núan siendo subvalorados y esta falta de percepción hace que estos problemas no sean reconocidos como enfermedades. Por ello, este trabajo tiene el propósito de evaluar la magnitud de este problema en los infantes menores de cinco años, en base a las Endes 1991/1992, con respecto a datos similares de las Endes 1996 y 2000, efectuando análisis por niveles de pobreza, según género, regiones geográficas y lugar de residencia (urbano/rural).

\section{MATERIALES Y MÉTODOS}

Se evaluó los indicadores antropométricos de acuerdo al patrón de referencia utilizado por el Instituto Nacional de Estadística Informática (INEI) peruano, definido por el Centro Nacional para las Estadísticas de la Salud de los Estados Unidos (NCHS) ${ }^{1}$ ). Se estableció como rango normal entre -1 y +1 desviación estándar (DE). El niño desnutrido, con $\mathrm{Z}$ menor a -2 DE y obeso/a para la relación peso/talla con $\mathrm{Z}$ mayor +2 .

Se consideró niños de ambos sexos con obesidad pediátrica (niños menores de dos años) u obesidad infantil (niños de entre dos y cinco años), así como departamento de proveniencia y área de residencia (rural o urbana).

Los años de estudios maternos aprobados fueron clasificados en los siguientes grupos: analfabeta, menor a 2 años de estudio; primaria, 2 a 6 años; secundaria, 7 a 12 años; y, superior, 13 a 18 años.

Los niveles de pobreza fueron establecidos por medio del método de necesidades básicas insatisfechas del INEI $\left(^{2}\right)$, que clasifica a la población en tres categorías $\left({ }^{3}\right)$ :

a) no pobres: hogares sin necesidades básicas insatisfechas (NBI);

b) pobres: hogares con una NBI; y,

c) extremadamente pobres: hogares con dos o más NBI. 
El análisis se realizó con el paquete estadístico SPSS y el EpiInfo versión 6,04d. En todos los casos, los resultados fueron considerados significativos con $p<0,05$. El análisis de regresión logística consideró las variables edad del niño en meses (E), género $(G)$, departamento de proveniencia (Dpto) y área de residencia (AR).

\section{RESULTADOS}

Las Tablas 1 y 2 presentan las características generales de la población estudiada en cuanto a puntaje $\mathrm{z}$ para peso/edad, talla/edad y peso/talla, edad promedio y distribución por género, clasificadas de acuerdo a las Endes.

La prevalencia de desnutrición crónica en los niños menores de 5 años disminuyó de $33 \%$, en el año 1992 , a $29 \%$, en el 2000 , valor cercano al 30\% hallado para el año 1999 , en el Censo de Talla para escolares $\left({ }^{4}\right)$.

En el grupo de niños entre 2 y 5 años, la desnutrición crónica disminuyó de 39\% (1992) a 32\% (2000), mientras el riesgo de desnutrición alcanzó el $31 \%$ para el último año (Figura 1).

La desnutrición global disminuyó de $12 \%$ (1992) a 9\% (2000), mientras el riesgo de desnutrición disminuyó de 31 a $29 \%$, para los niños entre dos y cinco años, cantidad que representa a más de la cuarta parte de estos niños.

La desnutrición aguda disminuyó de $2 \%$, en el año 92, a $1 \%$, en el 2000, mientras el sobrepeso y la obesidad ascendieron de $17 \%$ y $5 \%$, en el año 92 , a $19 \%$ de sobrepeso y $7 \%$ de obesidad, en el año 2000 (Figura 2). La Endes 2000 determinó un promedio nacional de desnutrición aguda de 1,45\% y

Tabla 1. Caracterización de niños menores de 2 años, según diferentes variables.

\begin{tabular}{|c|c|c|c|c|c|c|c|}
\hline \multirow[t]{2}{*}{ Variable } & \multicolumn{2}{|c|}{ Endes 1992} & \multicolumn{2}{|c|}{ Endes 1996} & \multicolumn{2}{|c|}{ Endes 2000} & \multirow{2}{*}{$\begin{array}{c}\text { Total } \\
\mathrm{N} \\
\end{array}$} \\
\hline & $\mathrm{N}$ & $\mathrm{x} \pm \mathrm{DE}$ & $\mathrm{N}$ & $\mathrm{x} \pm \mathrm{DE}$ & $\mathrm{N}$ & $\mathrm{x} \pm \mathrm{DE}$ & \\
\hline z Talla / Edad & 3177 & $-1,00 \pm 1,63$ & 6051 & $-0,95 \pm 1,62$ & 4567 & $-1,08 \pm 1,53$ & 13795 \\
\hline z Peso / Edad & 3177 & $-0,30 \pm 1,78$ & 6073 & $-0,35 \pm 1,40$ & 4567 & $-0,27 \pm 1,37$ & 13817 \\
\hline z Peso / Talla & 3094 & $0,28 \pm 1,25$ & 5929 & $0,33 \pm 1,26$ & 4463 & $0,47 \pm 1,23$ & 13486 \\
\hline Edad (meses) & 3177 & $11,24 \pm 6,76$ & 6104 & $11,70 \pm 6,72$ & 4567 & $11,90 \pm 6,77$ & 13848 \\
\hline Género & \multicolumn{2}{|c|}{$3177(100 \%)$} & \multicolumn{2}{|c|}{$6104(100 \%)$} & \multicolumn{2}{|c|}{$4567(100 \%)$} & 13848 \\
\hline Masculino & \multicolumn{2}{|c|}{$1609(50,6 \%)$} & \multicolumn{2}{|c|}{$3129(51,3 \%)$} & \multicolumn{2}{|c|}{$2346(51,4 \%)$} & 7084 \\
\hline Femenino & \multicolumn{2}{|c|}{$1568(49,4 \%)$} & \multicolumn{2}{|c|}{$2975(48,7 \%)$} & \multicolumn{2}{|c|}{2221 (48,6\%) } & 6764 \\
\hline
\end{tabular}

Tabla 2. Caracterización de niños entre 2 y 5 años según diferentes variables.

\begin{tabular}{|c|c|c|c|c|c|c|c|}
\hline \multirow[t]{2}{*}{ Variable } & \multicolumn{2}{|c|}{ Endes 1992} & \multicolumn{2}{|c|}{ Endes 1996} & \multicolumn{2}{|c|}{ Endes 2000} & \multirow{2}{*}{$\begin{array}{c}\text { Total } \\
\mathrm{N}\end{array}$} \\
\hline & $\mathrm{N}$ & $\mathrm{x} \pm \mathrm{DE}$ & $\mathrm{N}$ & $\mathrm{x} \pm \mathrm{DE}$ & $\mathrm{N}$ & $\mathrm{x} \pm \mathrm{DE}$ & \\
\hline z Talla / Edad & 4615 & $-1,60 \pm 1,32$ & 9015 & $-1,41 \pm 1,37$ & 7218 & $-1,46 \pm 1,37$ & 20848 \\
\hline z Peso / Edad & 4673 & $-0,78 \pm 1,13$ & 9110 & $-0,62 \pm 1,17$ & 7218 & $-0,59 \pm 1,16$ & 21001 \\
\hline z Peso / Talla & 4671 & $0,31 \pm 0,97$ & 9080 & $0,35 \pm 0,97$ & 7173 & $0,38 \pm 0,98$ & 20924 \\
\hline Edad (meses) & 4679 & $41,61 \pm 10,23$ & 9160 & $41,57 \pm 10,32$ & 7218 & $41,81 \pm 10,40$ & 21057 \\
\hline Género & \multicolumn{2}{|c|}{4679 (100\%) } & \multicolumn{2}{|c|}{$9160(100 \%)$} & \multicolumn{2}{|c|}{7218 (100\%) } & 21057 \\
\hline Masculino & \multicolumn{2}{|c|}{2390 (51,1\%) } & \multicolumn{2}{|c|}{$4537(49,5 \%)$} & \multicolumn{2}{|c|}{3575 (49,5\%) } & 10502 \\
\hline Femenino & \multicolumn{2}{|c|}{$5589(48,9 \%)$} & \multicolumn{2}{|c|}{$4623(50,5 \%)$} & \multicolumn{2}{|c|}{3643 (50,5\%) } & 13855 \\
\hline
\end{tabular}




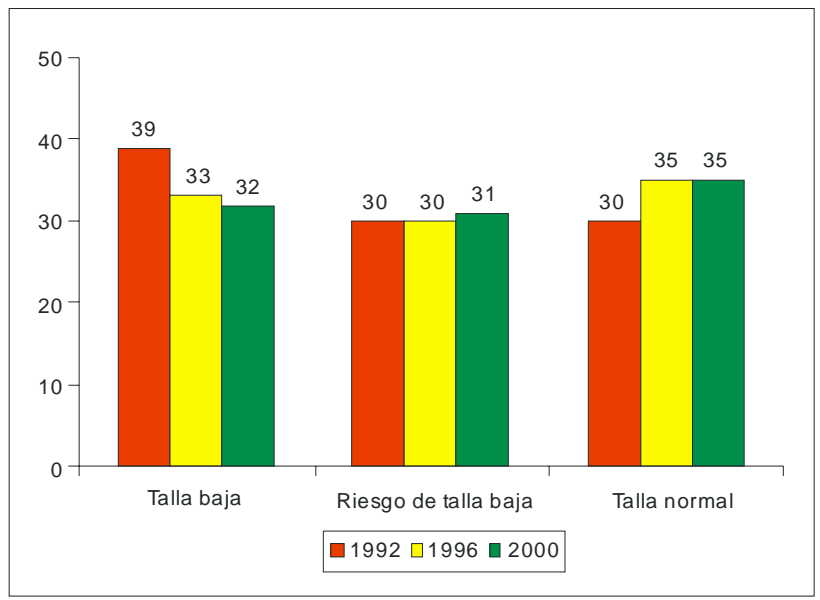

Figura 1. Estado nutricional de niños entre dos y cinco años, por indicador talla/edad.

un riesgo de desnutrición de $6,36 \%$, siendo los departamentos más afectados Ayacucho (3,41\%), Pasco (3,03\%), Madre de Dios $(2,46 \%)$ y Loreto $(2,2 \%)$.

En el grupo de niños entre dos y cinco años, la disminución de la desnutrición aguda fue más notoria (en $62,5 \%$ ), el riesgo de desnutrición se mantuvo alrededor de 5,30, mientras el sobrepeso (S) y la obesidad (O) se incrementaron paulatinamente, en $3,59 \%$ y $32,43 \%$, respectivamente. Mientras tanto, la prevalencia de obesidad encontrada para el año 2000 fue 4,9\%.

Los datos mostraron ligera mayor prevalencia de talla baja, riesgo de talla baja y desnutrición aguda en el sexo masculino y una leve mayor prevalencia de $\mathrm{S}$ y $\mathrm{O}$ en las niñas.

La distribución de la población infantil por grupos etáreos no presentó diferencias significativas. La desnutrición aguda fue más prevalente en los lactantes de 0 a 23 meses, siendo los niños de 48 a 59 meses los menos afectados, existiendo una tendencia decreciente a partir de $\operatorname{los} 12$ a 23 meses, sin variaciones significativas por año de estudio (Figura 3).

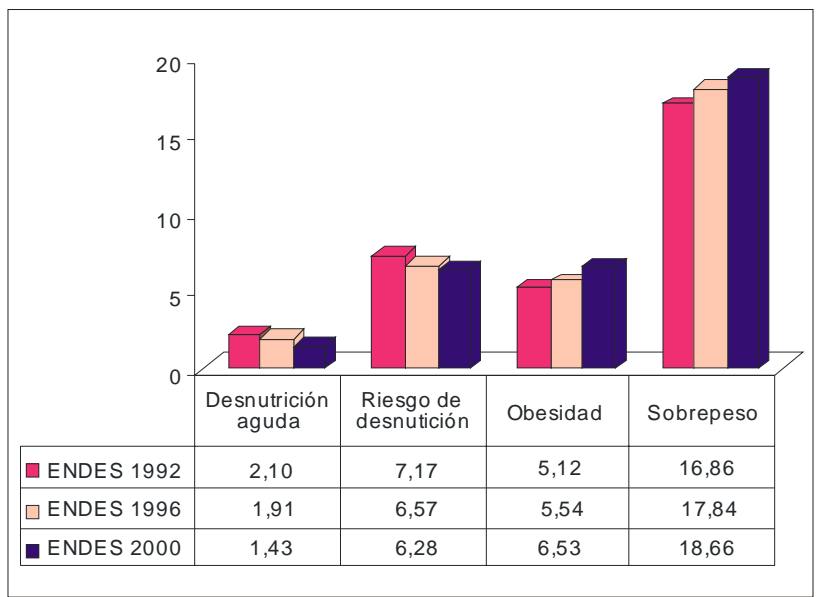

Figura 2. Estado nutricional de niños menores de cinco años de edad por indicador peso/talla.

Las mayores prevalencias de sobrepeso para los tres períodos se mostraron en ambos extremos de los grupos etáreos, niños de 0 a 11 meses y de 48 a 59 meses. Sin embargo, en el grupo de niños de 2 a 5 años, fueron los infantes mayores de 48 a 59 meses los que presentaron la mayor prevalencia de obesidad, siendo esta prevalen-

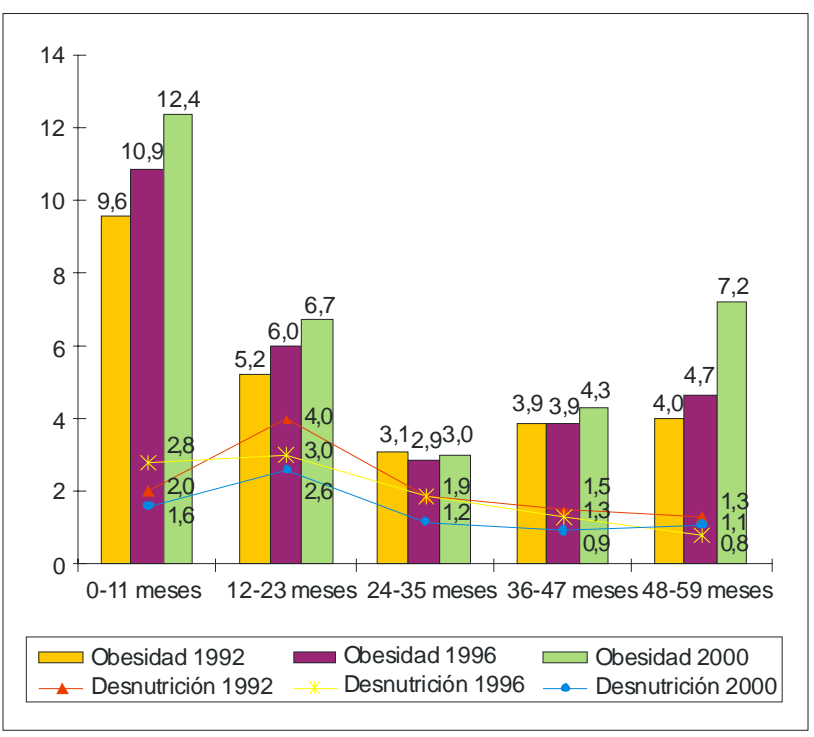

Figura 3. Desnutrición aguda y obesidad por grupos de edad. 
cia creciente en relación directa con la edad, a partir de los 24 meses. Durante todo el período, estas prevalencias son mucho mayores que las de desnutrición, para todos los grupos etáreos, incrementándose con el transcurso de los años.

En el año 1992, se observó mayor prevalencia de S y O (21\% y $7 \%)$ en el grupo de niños en situación de extrema pobreza, mientras en el 2000, aunque las mayores prevalencias se mantuvieron en ese grupo, no se presentaron diferencias significativas entre los tres niveles socioeconómicos, fluctuando en promedio alrededor de $18,53 \%$ de sobrepeso y $6,87 \%$ de obesidad. El porcentaje de niños con $\mathrm{S}$ y $\mathrm{O}$ no pobres disminuyó cuantiosamente, mientras se incrementaron los niños con $\mathrm{S}$ y $\mathrm{O}$, en el estrato de extremadamente pobres, disminuyendo a su vez considerablemente en el grupo de niños no pobres, demostrándose la asociación inversa existente entre el nivel socioeconómico y la obesidad, relacionándola directamente con los estilos de vida y los cambios dietéticos.

Se evidenció la importancia de la educación materna sobre el estado nutricional de los niños menores de cinco años de edad, mostrando que los niños de madres sin instrucción tienen mayor prevalencia de desnutrición crónica y mayor riesgo de talla baja, que los niños de madres con educación primaria y secundaria. Sin embargo, se determinó que, aunque el grupo de niños con madres con instrucción secundaria y superior presentaron ligera mayor prevalencia de $\mathrm{S}$ y $\mathrm{O}$, no se encontró relación marcada entre el grado de instrucción de la madre y el $\mathrm{S}$ y $\mathrm{O}$ en los niños menores de cinco años.

Pese a todo, la instrucción educativa nula o baja del padre fue un factor asociado a la desnutrición crónica (T/E) y, contrariamente, la instrucción superior incrementa la prevalencia de $\mathrm{S}$ y $\mathrm{O}$.
Los promedios nacionales de $\mathrm{S}$ y $\mathrm{O}$ para el año 2000 fueron $18,90 \%$ y 6,62\%, respectivamente, siendo este último valor muy cercano al $7 \%$ hallado en $1996\left(^{5}\right)$. Se determinó también variaciones intradepartamentos, presentándose Tacna $(41,49 \%)$, Moquegua $(29,60 \%)$ y Lima $(29,50 \%)$ como los departamentos más afectados por el sobrepeso, mientras estos mismos fueron los más afectados por la obesidad, en el siguiente orden: Tacna $(15,77 \%)$, La Libertad (12,87\%), Moquegua $(12,56 \%)$ y Lima $(11,21 \%)$, siendo este último valor similar al $12 \%$ encontrado para esta ciudad el $2002\left(^{6}\right)$.

Este estudio encontró además que existe mayor prevalencia de $\mathrm{S}$ y $\mathrm{O}$ en los departamentos de Tacna, Moquegua y Lima, con valores de $57 \%, 42 \%$ y $41 \%$, respectivamente, mientras Ucayali (16\%), San Martín $(15 \%)$ y Loreto (11\%) son los departamentos con menor prevalencia, aunque estos valores son similares a los reportados por Estados Unidos para niños de 6 a 19 años, entre 1988-2000 $\left({ }^{7}\right)$.

Para el grupo de niños de entre dos y cinco años, en el año 2000, los departamentos que ostentaron las mayores prevalencias de $\mathrm{S}$ y $\mathrm{O}$ fueron Ucayali $(28,42 \%)$, Tumbes $(28,03 \%)$ y Tacna $(27,63 \%)$.

El análisis por separado de $\mathrm{S}$ y $\mathrm{O}$ para este mismo grupo de niños, permitió observar curiosamente que los departamentos que exhibieron las mayores prevalencias de obesidad presentaron las menores prevalencias de sobrepeso. Así, para el año 2000, las mayores prevalencias de sobrepeso se presentaron en los departamentos de Arequipa (25\%), Ancash (23\%) y Cuzco (13\%) y las mayores prevalencias de obesidad en Tacna (19\%), Tumbes $(20 \%)$ y Ucayali $(21 \%)$

Sorprendentemente, de la misma forma, la ruralidad representó también un mayor riesgo para el $\mathrm{S}$ y $\mathrm{O}$, ya que si bien en el período analizado la menor prevalencia se 
presentó en los niños con $\mathrm{S}$ y $\mathrm{O}$ del área rural, ésta se ha incrementado con el correr de los años en $10,13 \%$, creciendo de $36,60 \%$, en el año 1992 , a $39,17 \%$, en el 96 , y $46,73 \%$, en el año 2000; mientras contrariamente en el área urbana la prevalencia viene descendiendo, de $63,40 \%$, en el año 92, a 53,27\%, en el 2000.

Al efectuar un análisis por área de residencia en los tres departamentos que presentaron la mayor prevalencia de obesidad, según la Endes 2000 (Tacna, Tumbes y Ucayali), encontramos resultados diferentes para cada uno de ellos. Mientras en Tacna es muy notoria la prevalencia de ambos problemas en el área urbana, en Tumbes no existe variación significativa de $\mathrm{S}$ y O por área de residencia, y en Ucayali no existe diferencia de sobrepeso por lugar de residencia; pero, en este caso, la obesidad se cuadruplica en el área urbana.

A nivel nacional, existen tendencias inversas entre el $\mathrm{S}$ y $\mathrm{O}$ y la desnutrición, mostrando que a lo largo del período evaluado, mientras el primero va incrementándose, el segundo ha ido disminuyendo notoriamente, respondiendo a las siguientes ecuaciones:

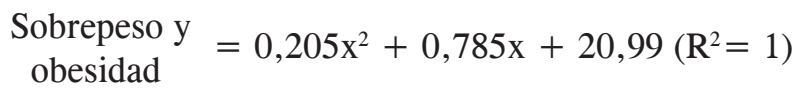

Desnutrición $=-0,78 x+10,047\left(R^{2}=0,9999\right)$

El sobrepeso y la obesidad nacionales generan líneas de tendencia crecientes, que van de $17 \%$ de sobrepeso, en los años 92/ 96 , a $19 \%$, en el año 2000 , y de $5,1 \%$ de obesidad a $5,5 \%$ y $6,5 \%$, respectivamente.

Si bien el sobrepeso y la obesidad nacionales generan líneas de tendencia crecientes, aparentemente a nivel departamental ocurre lo contrario. Por ejemplo, en Tacna la tendencia de $\mathrm{S}$ y $\mathrm{O}$ fue negativa para el segmento 1996/2000, mientras persiste la tendencia positiva creciente para los departamentos de Tumbes y Ucayali.
El análisis efectuado en los departamentos de Tacna, Lima y Moquegua comprobó que, aunque aparentemente el problema en conjunto de $\mathrm{S}$ y $\mathrm{O}$ en estos lugares ha ido disminuyendo, esto se debe a la leve disminución de sobrepeso y a su vez incremento de obesidad, problema aún más serio, con tendencia positiva creciente.

Por otro lado, las proyecciones nacionales de $\mathrm{S}$ y $\mathrm{O}$ para niños menores de 5 años son alarmantes, con tendencia creciente y siempre superiores a las proyecciones de desnutrición para los niños de la misma edad.

Para los niños entre dos y cinco años, las proyecciones de los promedios departamentales de sobrepeso van en notoria disminución, lo que sería alentador si no ocurriera lo contrario con las proyecciones de obesidad para el mismo grupo de niños, que llegan hacia el año 2004 a sobrepasar el $50 \%$ de obesidad en el caso de Tumbes y Ucayali y, a partir de 2008, supera el $38 \%$ en todos los departamentos analizados (Figura 4).

Finalmente, la regresión logística aplicada a cada una de las variables indica que casi todas, exceptuando el género, tienen

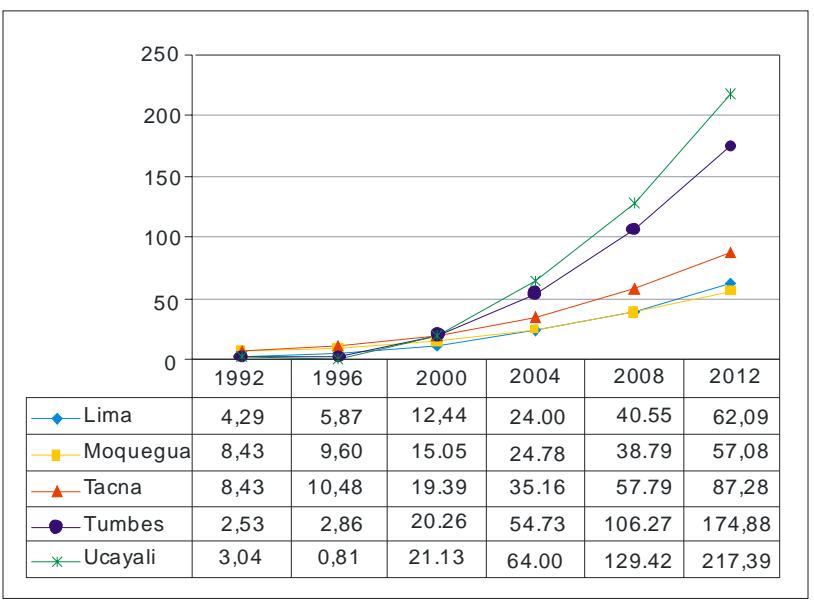

Figura 4. Proyección departamental de obesidad para niños de 2 a 5 años. 
efecto estadísticamente significativo y por tanto afectan el $\mathrm{S}$ y $\mathrm{O}$ en los niños entre dos y cinco años. Sin embargo, la ecuación de regresión logística binaria, mediante la que podemos expresar el $\mathrm{S}$ y $\mathrm{O}$ en función de las todas las variables estudiadas, fue la siguiente:

$$
\begin{aligned}
\mathrm{S} \text { y } \mathrm{O}(\mathrm{SO})= & -5,759+0,027 \mathrm{E}-0,467 \mathrm{AR}+0,081 \mathrm{IM} \\
& +0,028 \mathrm{G}-0,002 \mathrm{Dpto}+2,284 \mathrm{NP}
\end{aligned}
$$

El valor $p$ de la prueba aplicada al modelo resultó significativo. Del mismo modo, el análisis de regresión logística general determinó que las variables más significativas como factor de riesgo para el SO, mostrando coeficientes de regresión que presentaron valores de $\mathrm{z}$ con probabilidades menores de 0,05, fueron la edad, el área de residencia y los niveles de pobreza.

Considerando estos resultados, la corrida final resultó siendo la siguiente:

$$
\begin{aligned}
\mathrm{SO}= & -5,5211+0,027 \text { Edad }-0,535 \text { Área de resi- } \\
& \text { dencia }+2,286 \text { Nivel de pobreza }
\end{aligned}
$$

\section{DISCUSIÓN}

Se determinó que, aunque persiste el problema de desnutrición en los niños peruanos menores de cinco años; los problemas de malnutrición por exceso, como el sobrepeso y la obesidad ( $\mathrm{S}$ y $\mathrm{O}$ ), alcanzan actualmente valores considerablemente elevados, siendo los factores de riesgo determinantes el nivel de pobreza, la edad y el área de residencia, quedando la instrucción materna, el género y departamento de proveniencia descartados como variables influyentes.

Es importante remarcar que, los resultados encontrados en este estudio difieren en varios casos de los señalados por las Endes, debido a que se refieren a niños entre 1 y 59 meses; aun así, las tendencias presentadas en ambos estudios son las mismas.
La desnutrición crónica aquí determinada indica que, entre el período 1992/2000 hubo una mejora nutricional, que se reflejó en una ligera mejor talla en los niños menores de cinco años. Sin embargo, el $32 \%$ hallado aún es elevado, esto posiblemente debido a la presencia de repetidas infecciones en los primeros años de vida, la falta de agua en el hogar y la talla baja del padre, que actúan como factores de riesgo de talla baja en el Perú $\left({ }^{8,9}\right)$. También, se considera la ruralidad, posesión de bienes, hacinamiento, sector económico del jefe de hogar y nivel educativo del jefe de hogar $\left({ }^{10}\right)$. Es por esta complejidad del problema que, se recomienda que el estudio de la talla baja deba individualizarse para cada población $\left({ }^{9}\right)$. Además, por definición propia de talla baja, el $3 \%$ de la población tendrá talla baja y en ese sentido se presentan complicaciones debidas principalmente a las variantes regionales $\left({ }^{11}\right)$, originadas por la distribución geográfica y situación de pobreza del país $\left({ }^{4,12,13}\right)$.

Por otro lado, la desnutrición global es un indicador compuesto por la talla para la edad y el peso para la talla, por lo que el indicador agrupa a niños que pueden ser normales o muy delgados. Por eso, este índice no es muy exacto, debido a que el niño que es pequeño para su edad por razones genéticas o seculares puede aparecer como desnutrido sin serlo y niños con talla normal y peso bajo para la talla pueden pasar desapercibidos $\left({ }^{14}\right)$. Consecuentemente, en estudios transversales como este, el índice peso/edad es menos útil que los otros indicadores; tiene más utilidad en el campo clínico y en evaluaciones de seguimiento individual $\left({ }^{14}\right)$. Por ello, los valores relativamente bajos encontrados en este estudio podrían darse porque los casos graves de desnutrición en comunidad son, en general, menos frecuentes que los casos de desnutrición leve o moderada y éstos no pueden ser detectados con facilidad en una sola evaluación, sin contar con referencias previas de cada niño. 
Los resultados hallados muestran también que, en niños menores de cinco años la ruralidad significó un mayor riesgo, tanto de talla baja y desnutrición aguda como de sobrepeso y obesidad. Aunque parezca contradictorio, esto es similar a lo encontrado en Chile $\left({ }^{15}\right)$, confirmando que las sociedades menos desarrolladas y los ambientes rurales tienden a desplegar una asociación más positiva entre el sobrepeso y el estrato social $\left(^{5}\right)$.

La prevalencia de obesidad en niños menores de cinco años ha permanecido alrededor de $6 \%$, en el período estudiado $(5,6 \%$, en $1992,5,2 \%$, en 1996 , y $6,6 \%$, el año 2000), valores similares a los encontrados en Paraguay $(5,3 \%)$ y Uruguay $(6,2 \%)$ y ligeramente menores a los de Chile $(7,1 \%)$ y Argentina $(7,3 \%)$, que son los países latinoamericanos más afectados $\left({ }^{16}\right)$, siendo Chile uno de los países en que la obesidad infantil ha crecido a una velocidad de las más grandes en el mundo.

La obesidad $(4,9 \%)$ hallada en niños entre dos y cinco años fue mayor al $4,6 \%$ del promedio latinoamericano $\left({ }^{17}\right)$ y ligeramente menor al $7,7 \%$ ostentado por Chile para niños de la misma edad, para el mismo año 2000.

El género no ha sido encontrado como factor de riesgo, racionalmente tampoco ofrece causalidad directa, pero las costumbres sociales que influyen en la crianza de las niñas hacen que se considere como factor asociado $\left({ }^{18}\right)$.

Es innegable que, las estrategias familiares de vida son determinantes de la ocurrencia de malnutrición. Investigaciones previas han encontrado que el principal factor protector del estado nutricional de los niños en situación de pobreza es el mejor nivel educativo de la madre, constituyendo seis años de estudio en promedio el punto de corte para observar una menor prevalencia de desnutrición en los niños menores de cinco años de edad $\left({ }^{19}\right)$.
La relación directa hallada en este estudio, entre el S y $\mathrm{O}$ con el grado de instrucción del padre, muestra que en la mayoría de los casos esto se relaciona con una mejor situación económica y con un ascenso en el nivel de vida, caracterizado por el consumo de mayor cantidad de alimentos de todo tipo y acceso a mejores servicios, lo que genera cambios nocivos en los modelos nutricionales y la actividad física $\left({ }^{20}\right)$. A la vez, puede ocurrir que los estratos bajos pueden percibir que los cuerpos más pesados son más saludables para el trabajo y protegen la parte económica, influyendo en conductas asociadas, así como en la dieta y la actividad física $\left(^{5}\right)$; obligando a reconocer la existencia del problema de obesidad en condiciones de pobreza, dentro de un contexto integral en el que interactúa con otros problemas nutricionales y con una serie de factores sociales, económicos y culturales $\left({ }^{21}\right)$, lo que es entender de inicio la transición nutricional por la que atraviesa nuestro país.

A esto hay que adicionarle los problemas internos. A nivel departamental, la distribución de la obesidad está afectada por la población y puesto que la estructura poblacional (distribución y densidad) de nuestro país es muy variada $\left({ }^{22}\right)$, los valores difieren de las prevalencias por departamento. En el caso de Lima, por ejemplo, siendo la ciudad más poblada del Perú, contaba el año 2000 con el mayor número de niños con $\mathrm{S}$ y $\mathrm{O}$ del país $(18,7 \%$ y $6,5 \%)$, y a su vez representaban en total el $14 \%$ de los niños con S y O de todo el país.

Las altas prevalencias de obesidad en Tacna, Tumbes y Ucayali en niños entre dos y cinco años amerita realizar investigaciones exhaustivas, que permitan detectar si existen actualmente algunos factores que estén determinando un mayor riesgo de $\mathrm{S}$ y $\mathrm{O}$ en estas regiones. No obstante, existen razones que hacen suponer que, gran parte de estas variaciones se explican porque cada 
uno de los departamentos pertenece a una zona geográfica distinta, lo que genera la diversidad de historias, costumbres y economías, así como la variada disponibilidad de alimentos existente en cada espacio regional, que influye tanto en los hábitos alimenticios como en la situación económica de cada departamento; ello origina marcadas diferencias de pobreza y capacidades de adquisición entre ellos. Así, la agrupación de los departamentos del país en estratos de pobreza según necesidades básicas insatisfechas (NBI) clasifica a Tacna dentro de los departamentos del primer estrato económico, a Tumbes dentro de los del tercero y a Ucayali dentro de los del cuarto estrato, hecho que determina el poder adquisitivo de sus poblaciones, situación nutricional y niveles de sobrepeso ${ }^{(22}$.

$\mathrm{Y}$, al margen de su capacidad adquisitiva, debemos considerar también la amplia y polarizada variedad étnica y cultural, especialmente de las comunidades andinas y amazónicas peruanas $\left({ }^{22}\right)$, que hace que varíen igualmente sus rasgos genéticos como su cultura nutricional, que influyen directamente en el nivel nutricional de sus niños.

También, influyen la disponibilidad de alimentos en cada zona, así como a las diferentes labores que se realiza por área de residencia en cada una de las regiones que, por su misma geografía, generan labores disímiles. La OPS $\left({ }^{20}\right)$ opina al respecto que los actuales sistemas alimentarios basados en un enfoque agropecuario industrial permiten disponer de casi todos los tipos de comida durante todo el año, independientemente de la estación y del lugar de residencia. Además, el rápido crecimiento del suministro de alimentos procesados y de alto contenido graso y energético a todo nivel ha contribuido a la mejor disponibilidad de alimentos, pero no necesariamente ha mejorado la calidad nutricional general de los alimentos $\left({ }^{20}\right)$.

\section{REFERENCIAS BIBLIOGRÁFICAS}

1. Barrera G. Estándares antropométricos para evaluación del estado nutritivo. Instituto de Nutrición y Tecnología de los Alimentos (INTA). Santiago: Universidad de Chile; 2001.

2. Instituto Nacional de Estadística e Informática del Perú (INEI). Programa de Encuestas de Demografía y Salud (DHS) 1992/ 1996/2000. Macro International Inc. Calverton MD EEUU. Encuesta de Demografía y de Salud Familiar (ENDES). Lima: INEI; 2001.

3. Instituto Nacional de Estadística e Informática (INEI). Salud productiva, pobreza y condición de vida en el Perú [monografía en Internet]. Lima: INEI; 1994 [citado 04 Mayo 2004]. Disponible en: http://www.inei.gob.pe/biblioineipub/ bancopub/Est/Lib0078/S04.htm

4. Ministerio de Agricultura (MINAG). Informe Nacional sobre la Seguridad Alimentaria en el Perú. Capítulo II: La situación de inseguridad alimentaria en el Perú [monografía en Internet]. Lima: MINAG; 2002. Disponible en: http:// www.portalagrario.gob.pe

5. Jacoby E, Goldstein J, Lopez A, Nunez E, Lopez T. Social class, family, and life-style factors associated with overweight and obesity among adults in Peruvian cities. Prev Med. 2003;37(5):396-405.

6. Guevara C. Desarrollo puberal, índice de masa corporal y talla final en niñas obesas atendidas en el Instituto de Salud del Niño [Tesis de Especialista en Pediatría]. Lima: Facultad de Medicina Humana, Escuela de Post-Grado. Universidad Nacional Mayor de San Marcos; 2002.

7. Troiano RP, Flegal KM, Kuczmarski RJ, Campbell SM, Johnson CL. Overweight prevalence and trends for children and adolescents. The National Health and Nutrition Examination Surveys, 1963-1991. Arch Pediatr Adolesc Med. 1995;149:1085-91.

8. Andraca I, Castillo M, Cortés F. Factores de riesgo para talla baja en escolares de nivel socioeconómico bajo. Caracterización biomédica, social y cognitiva. Santiago: Instituto de Nutrición y Tecnología de los Alimentos (INTA), Universidad de Chile; 1993.

9. Tanner JM, Isrealsohn WJ. Parent-child correlations for body measurements of children between the ages of one month and seven years. Ann Hum Genet. 1963;26:245.

10. Montes C, Segura L, Haustein D, Baltazar G, Vigil N. Marcadores de la desnutrición crónica en áreas urbana y rural de cinco corredores económicos. Lima: Prisma; 2000.

11. Dorantes-Álvarez L, García L. Evaluación del tratamiento de la talla baja constitucional con hormona de crecimiento obtenida de células de mamífero. Bol Med Hosp Infant Mex [artículo en Internet]. 1995;52:431-4 [citado 05 Julio 2004]. Disponible en: http://www.drscope.com/privados/pac/ pediatria/pal2/index.html

12. Cortez, R. La nutrición de los niños en edad pre-escolar. Centro de investigación de la Universidad del Pacífico [monografía en Internet]. Enviado al consorcio de 
investigación económica y social como parte de la serie de investigaciones auspiciadas por el IDRC-CIES 2001-2002. Lima: Universidad del Pacífico; 2003 [citado 28 Mayo 2004]. Disponible en: http://www.consorcio.org/CIES/html/ pdfs/Pm0117.pdf

13. Gajate G, Inurretegui M. Programa Vaso de Leche: ¿Mejora la desnutrición crónica infantil?. Análisis de Políticas. Grupo Análisis para el Desarrollo (GRADE). Boletín del Consorcio de Investigación Económica y Social. (5);2003.

14. Frenk S. Síndrome de mala nutrición: Desnutrición [monografía en Internet]. Academia Mexicana de Pediatría. Programa de Actualización Continua en Pediatría (PAC P1). 1ra Edición. México: Edit. Intersistemas, S.A. de C.V. PAC. Libro 2, Parte A. Pag. 27 a la 38. [citado 02 Julio 2004]. Disponible en: http://www.drscope.com/privados/ pac/pediatria/pal2/sindrome.htm

15. Ministerio de Planificación y Cooperación (Mideplan). Encuesta Casen. Gobierno de Chile. Santiago: Mideplan; 1998.

16. Red de Cooperación Técnica sobre Sistemas de Vigilancia Alimentaria y Nutricional (Sisvan). Situación Alimentaria y Nutricional en América Latina. Magnitud y Tendencias. Buenos Aires: Sisvan; 2000.

17. Amigo H. Obesidad en el niño en América latina: situación, criterios de diagnóstico y desafíos. Departamento de Nutrición, Facultad de Medicina, Universidad de Chile. Cad saúde pública [artículo en Internet]. 2003;19(supl 1) [citado 01 junio 2004]:163-170. Disponible en: http:// www.scielo.br/scielo.php
18. Cedraz L, Carvalho F. Family income and child malnutrition in the coast of camacari, Brazil. Arch Latinoam Nutr. 1990;40(3):323-32.

19. Sanabria, MC. Determinantes del estado de salud y nutrición de niños menores de 5 años en situación de pobreza, Paraguay EIH 2000/01. Asunción, Paraguay; 2002.

20. Organización Panamericana de la Salud, Organización Mundial de la Salud. Obesidad, alimentación y actividad física. 37.a Sesión del subcomité de planificación y programación del comité ejecutivo. Washington, D.C.: OPS-OMS; 2003.

21. Amador M, Rodríguez-Ojea A. Seminario-taller sobre obesidad y pobreza en América Latina. Rev Cubana Aliment Nutr. 1996;10(1):49-52.

22. Oficina General de Epidemiología, Oficina Ejecutiva de Análisis de Situación de Salud y Tendencias (ASIS). Condicionantes y determinantes de la situación de salud. Capitulo I. Lima: ASIS; 2001.

Manuscrito recibido el 21 de agosto de 2006 y aceptado para publicación el 21 setiembre de 2006.

\author{
Correspondencia: \\ Mg.Sc. Luz Bullón Camarena \\ Universidad Agraria La Molina \\ Av. La Molina s/n. Lima 12, Perú \\ Correo-e:luz@lamolina.edu.pe
}

